\title{
pKAl: a fast and interpretable deep learning approach for accurate electrostatics-driven pKa predictions
}

Pedro Reis ( $\sim$ pdreis@ciencias.ulisboa.pt)

BioISI - Biosystems \& Integrative Sciences Institute https://orcid.org/0000-0003-3563-6239

Marco Bertolini

Bayer AG

Floriane Montanari

Bayer AG

\section{Walter Rocchia}

Istituto Italiano di Tecnologia https://orcid.org/0000-0003-2480-7151

Miguel Machuqueiro

BiolSI - Biosystems \& Integrative Sciences Institute https://orcid.org/0000-0001-6923-8744

Djork-Arné Clevert

Bayer AG

Article

Keywords: pKa shifts, deep learning, accuracy

Posted Date: October 5th, 2021

DOI: https://doi.org/10.21203/rs.3.rs-949180/v1

License: (1) This work is licensed under a Creative Commons Attribution 4.0 International License.

Read Full License 
pKAl: a fast and interpretable deep learning

. approach for accurate electrostatics-driven $\mathrm{p} K_{\mathrm{a}}$

predictions

\author{
Pedro B.P.S. Reis ${ }^{1,2,3,{ }^{*}}$, Marco Bertolini ${ }^{1}$, Floriane Montanari ${ }^{1}$, Walter Rocchia ${ }^{3}$, Miguel \\ Machuqueiro $^{2,}$, and Djork-Arné Clevert ${ }^{1, ~}$

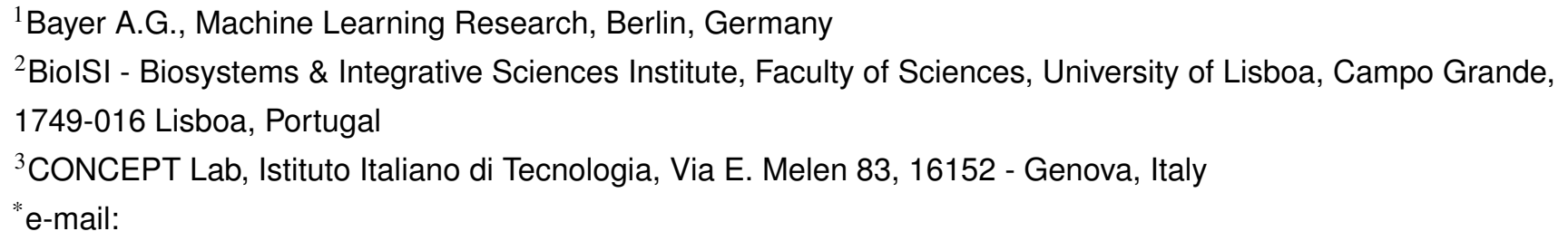

11 ABSTRACT

The $\mathrm{p} K_{\mathrm{a}}$ values of ionizable residues influence protein folding, stability and biological function. The $\mathrm{p} K_{\mathrm{a}}$ in bulk water is known for all residues, however, in a protein environment, it can significantly be affected by confinement and electrostatics. Existing computational methods to estimate $\mathrm{p} K_{\mathrm{a}}$ shifts rely on theoretical approximations and lengthy computations. Furthermore, the amount of experimentally determined $\mathrm{p} K_{\mathrm{a}}$ values is still very limited, hindering the development of faster machine learning-based methods. In this work, we use a data set of 6 million $\mathrm{p} K_{\mathrm{a}}$ shifts - determined by PypKa, a continuum electrostatics method - to train deep learning models that are shown to rival the physics-based predictor. On $\sim 750$ experimentally determined data points, our model displays the best accuracy and it is the only one that breaks the $1 \mathrm{p} K$ unit RMSE barrier of this considerably difficult test set. Although trained using a very simplified view of the surroundings of the titratable group (namely, atom types and distances to other titratable groups within a given radius), the models are shown to assign proper electrostatic charges to chemical groups, to keep the known correlation between solvent exposure and $\mathrm{p} K_{\mathrm{a}}$ shift magnitude, and to grasp the importance of close interactions, including hydrogen bonds. Inference times allow speedups of more than 1000 times faster than physics-based methods, especially for large proteins. By combining speed, accuracy and a reasonable understanding of the theoretical basis for $\mathrm{p} K_{\mathrm{a}}$ shifts, our models provide a game-changing solution for fast estimations of macroscopic $\mathrm{p} K_{\mathrm{a}}$ from ensembles of microscopic ( $\mathrm{p} K_{\text {half }}$ ) values as well as for many downstream applications such as molecular docking and constant-pH molecular dynamics simulations. 
Many biological processes are triggered by changes in the ionization state of key amino acid side-chains ${ }^{1,2}$. Experimentally, the titration behavior of a molecule can be measured using potentiometry or by tracking free energy changes across a $\mathrm{pH}$ range. For individual sites, titration curves can be derived from infrared or NMR spectroscopy. Detailed microscopic information can be quickly and inexpensively obtained with computational methods, and several in silico $\mathrm{p} K_{\mathrm{a}}$ calculations have become widely used to provide insights about structural and functional properties of proteins ${ }^{3-5}$.

In Poisson-Boltzmann-based (PB) methods, the solvent is implicitly described while proteins are represented by point charges in a low dielectric medium ${ }^{3,4,6,7}$. These continuum electrostatics (CE) methods assume that the $\mathrm{p} K_{\text {half }}$ (the proton binding affinity for a chemical group in a given conformation) is a good estimate for the macroscopic $\mathrm{p} K_{\mathrm{a}}$ value. This assumption holds when the protein structure is sufficiently representative of the conformational ensembles corresponding to both protonation states. Experimentally determined structures exhibit conformations at a minimum energy state, which, in turn, is related to a specific protonation state. However, biomolecular systems can explore different energy basins, which may exhibit alternative protonation states. Energy minima can be affected by experimental conditions, such as temperature, ionic strength and $\mathrm{pH}$. Inaccuracies in $\mathrm{p} K_{\mathrm{a}}$ predictions due to limited conformational rearrangements can be reduced by increasing the protein dielectric constant from its default value (2-4), which only accounts for electronic polarisation. The dielectric constant can be used as an empirical parameter mimicking the effect of the response mechanisms to the local electric field that are not explicitly taken into account in the model ${ }^{8-12}$. A more computationally expensive approach is to explicitly include protein motion by sampling conformers via Monte Carlo (MC) or molecular dynamics (MD) simulations and applying conformational averaging ${ }^{4,13-15}$. Finally, by coupling the sampling of protonation states at given $\mathrm{pH}$ and conformations, constant-pH MD methods ${ }^{16-20}$ provide greater insight into $\mathrm{pH}$-dependent processes ${ }^{21-25}$.

As larger data sets of experimental $\mathrm{p} K_{\mathrm{a}}$ values have become available, a new class of purely empirical methods has been developed. These models rely on statistical fits of empirical parameters weighting the different energetic contributions into simplified functions. PROPKA ${ }^{5}$ is arguably the most popular of such methods ${ }^{26}$, and has been shown to perform competitively even when compared to higher-level theory methods ${ }^{6,27}$. The empirical methods are much faster than the physics-based ones although at the cost of providing less microscopic insights, and their predictive power is unknown on mutations and/or proteins dissimilar to those composing the training set.

The accuracy of most predictors is bound to the estimation of the same quantity, the so-called $\Delta \mathrm{p} K_{\mathrm{a}}$. 
This is the free energy of transferring the ionizable residue from the solvent to the protein, compared to its neutral counterpart. Since $\mathrm{p} K_{\mathrm{a}}$ values for all amino acids in water have been experimentally determined, the $\mathrm{p} K_{a}^{\text {solvent }}$ term can be fixed and, in practice, it can also be adjusted to incorporate systematic errors. The $\Delta \mathrm{p} K_{\mathrm{a}}$ can be regarded as a sum of mostly electrostatic contributions stemming from the residue microenvironment. Therefore, an accurate prediction of $\mathrm{p} K_{\mathrm{a}}$ values for a given conformation requires a correct description of the residue interactions with the surrounding protein charges and with the solvent.

At their core, deep learning (DL) models are complex non-linear empirical functions fitted to best map input variables to output properties. Considering chemical properties, such as $\mathrm{p} K_{\mathrm{a}}$ values, which are dictated by molecular configurations, and provided that enough examples are presented, it is possible to train a model to map this relationship without the need to solve non-linear equations in 3D or to sort through the massive space of possible states.

In this paper, we have developed two DL-based $\mathrm{p} K_{\mathrm{a}}$ predictors: $\mathrm{pKAI}$ and $\mathrm{pKAI}+$, for $\mathrm{p} K_{\text {half }}$ and experimental $\mathrm{p} K_{\mathrm{a}}$ values, respectively. These models have been trained on a database with $\sim 6$ million $\mathrm{p} K_{\mathrm{a}}$ values estimated from $\sim 50$ thousand structures using a continuum electrostatics method, $\mathrm{PypKa}^{6}$. pKAI+ displays an unrivaled performance at predicting experimental $\mathrm{p} K_{\mathrm{a}}$ values on a $\sim 750$ members data set. Also, pKAI exhibits an accuracy comparable to the PB-based predictor used to generate the training set while being approximately $10-1000 \times$ faster. As we will show in this work, these are simple and interpretable methods that are able to implicitly model most of the required energetic contributions such as Coulomb interactions, desolvation and hydrogen-bonding. Therefore, the presented models feature the best characteristics of CE-based methods - accuracy and interpretability - with the speed provided by empirical approaches.

\section{Results}

The main goal of $\mathrm{pKAI}$ is to mimic the $\mathrm{p} K_{\mathrm{a}}$ predictive ability of Poisson-Boltzmann-based methods with a significant computational performance improvement. Our training set is comprised of $\mathrm{p} K_{\mathrm{a}}$ values calculated using PypKa on a large number of proteins taken from the Protein Data Bank ${ }^{28}$. An elaborate data split was performed to minimize data leakage from the training set to the validation and test sets (see Methods). pKAI was designed to be a simple and interpretable model using the minimum structural features that still capture the electrostatic environment surrounding the titratable residue. The model has been trained on $\Delta \mathrm{p} K_{\mathrm{a}}$ values rather than on absolute values. The $\mathrm{p} K_{\mathrm{a}}$ shift is in fact a more appropriate quantity to learn, less dependent on the chemical peculiarities of individual amino-acids and more sensitive to the local electrostatic environment. For example, residues that share a common side-chain chemical 
group (such as glutamate and aspartate sharing a carboxylic acid) are influenced by the same environment in a similar way.

We wanted our model to capture the electrostatic dependence between the environment of a residue and its consequent $\mathrm{p} K_{\mathrm{a}}$ shift while keeping the input layer as small as possible (see Methods). By ignoring all carbon and hydrogen atoms, we are greatly reducing the dimensionality of our input layer, while retaining most of the information regarding charged particles. There is of course a significant loss of topological information, although much can be inferred from the positions of the included atoms. In fact, there is no performance gain when adding solvent exposure measurements (e.g. solvent accessible surface area, residue depth) to the environment embedding. Considering that solvent exposure entails topological information and that the model is not able to extract additional information from it, we conclude that it was already estimating, to some degree, these molecular properties (see Model Explainability subsection).

\section{pKAl: predicting theoretical $\mathrm{p} K_{\mathrm{a}}$}

The performance of the model on the test set is reported in Table S1 and Figure 1A. The null model used for comparison consists of the reference $\mathrm{p} K_{\mathrm{a}}$ value in water for each residue type, and corresponds to 0 in the $\Delta \mathrm{p} K_{\mathrm{a}}$ scale. Overall, pKAI reproduces the Poisson-Boltzmann-based data with a MAE value of 0.31 and a RMSE of 0.52 , which is approximately half of the errors observed for most experimental $\mathrm{p} K_{\mathrm{a}}$ predictors ${ }^{6,29}$. However, in this case, we are only predicting theoretical values with a well-defined relation between structure and $\mathrm{p} K_{\text {half }}(\mathrm{p} K$ value of a single conformation). This relation is significantly more complex when dealing with experimental $\mathrm{p} K_{\mathrm{a}}$ values since the interactions present in the structure should be weighted according to their occurrence probability at equilibrium. The performance of pKAI is impressive considering the high complexity of the dependence between $\mathrm{p} K_{\mathrm{a}}$ and the site electrostatic environment, illustrated by the high RMSE value of the Null model (1.89). Some residues are easier to predict (e.g. LYS and termini residues) while others are more challenging (e.g. CYS and TYR). This can be explained by their solvent exposure distribution (Figure 1B): well-solvated residues exhibit small $\Delta \mathrm{p} K_{\mathrm{a}}$ values while more buried ones are more affected by the desolvation effect and establish more interactions with other residues causing their $\mathrm{p} K_{\mathrm{a}}$ values to shift. There is a clear dependency between the solvent exposure of a residue, its $\Delta \mathrm{p} K_{\mathrm{a}}$ value and the prediction difficulty (Figure $\mathrm{S} 1$ ). The excellent performance of pKAI is also demonstrated by the fact that most predictions (81.2\%) exhibit an error below $0.5 \mathrm{pK}$ units, which is a sufficient for most use cases.

The main motivation for developing DL models is the potential speedup. Since continuum electrostatics (CE) $\mathrm{p} K_{\mathrm{a}}$ estimations need to sample thermodynamic equilibrium microstates, several iterative simulations have to be performed on each protonation state and environment of every residue. On the other hand, 

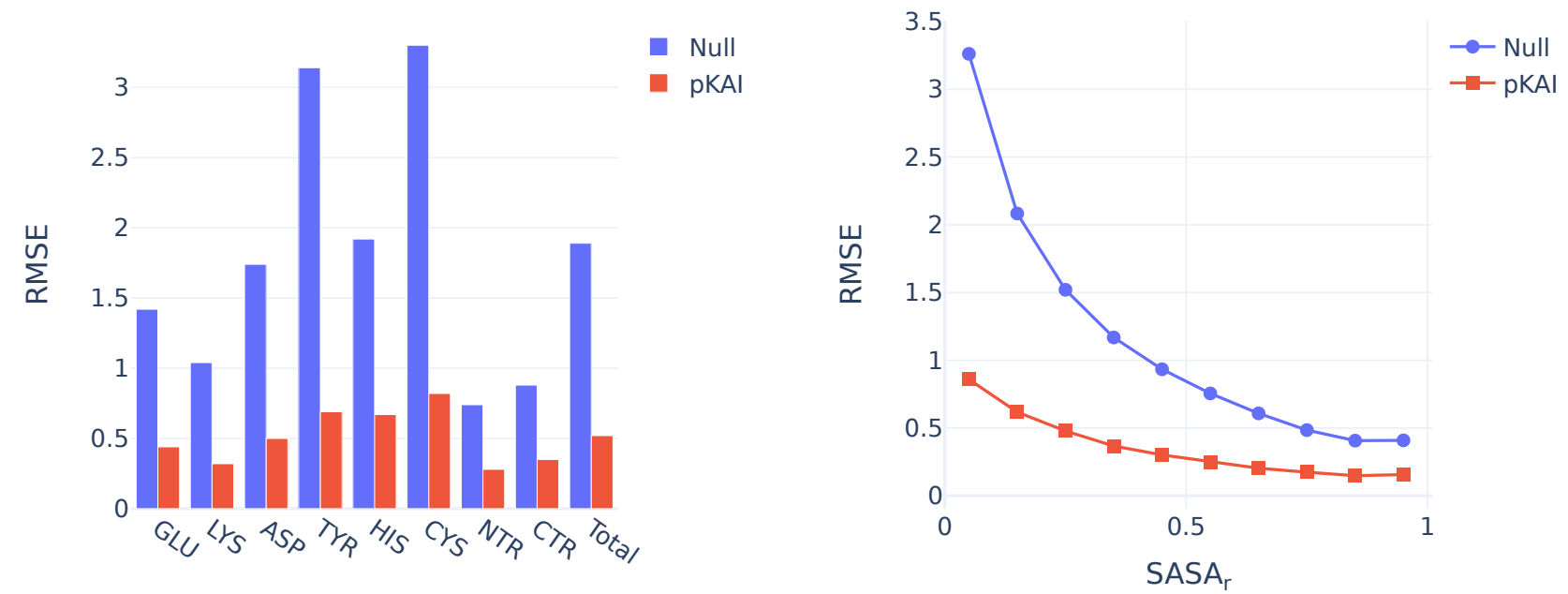

a)

b)
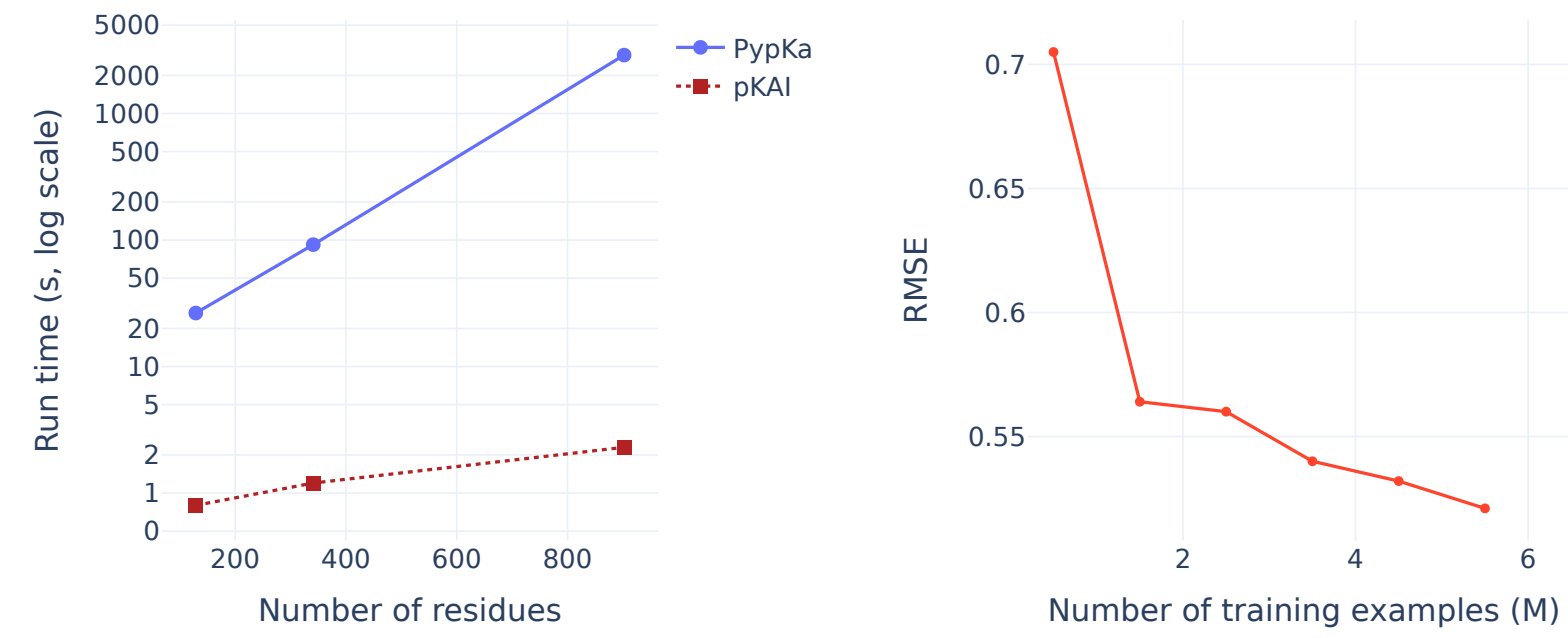

c)

d)

Figure 1. A) Comparison between Null model and pKAI RMSE values (values shown in Table S1). The Null model is defined as the $\mathrm{p} K_{\mathrm{a}}$ values of the residues in water taken from reference 30. B) Performance at predicting $\mathrm{p} K_{\text {half }}$ values dependency on the magnitude of solvent exposure (SASA). The calculations were performed for pKAI and Null model using the PypKa predictions as reference. C) Execution time comparison between PypKa and pKAI (values shown in Table S2). This benchmark was executed on a machine with a single Intel Xeon E5-2620 processor. D) Effect of the size of the training set in the model performance on the validation set. 
pKAI merely needs to apply its learned function over each residue and, as such, is remarkably faster (Figure 1C). Moreover, the convergence of the $\mathrm{CE}$ simulations is harder to achieve as the protein size increases. Consequently, in PypKa, as the protein size increases, so does the time required to estimate each $\mathrm{p} K_{\mathrm{a}}$ value. In contrast, the run time of $\mathrm{pKAI}$ 's DL model has a different dependence on the protein size. Since the bigger is the protein the larger is the amount of calculations that can be performed simultaneously, then the less significant becomes the model loading cost and the faster the average per-residue execution time. This results in sublinear scaling performance and in a speedup over its CE counterpart that can exceed over a thousand times. As such, pKAI is a particularly valuable tool for dealing with very large systems with thousands of residues where the only added computational cost stems from the prepossessing of the structure.

Another important factor contributing to the high accuracy obtained is the considerable size of the training set. Despite using the largest repository of experimental protein structures and the largest $\mathrm{p} K_{\mathrm{a}}$ database available ${ }^{28}$, we show that there is still a correlation between the number of examples in the training set and the accuracy of the model (Figure 1D). This indicates that our model can still be improved by providing further examples of $\mathrm{p} K_{\mathrm{a}}$ values. To avoid limiting the scaling rate by the availability of new experimental protein structures, we can generate new and uncorrelated protein structures using conformational sampling methods, such as MD and MC. Another advantage of using computational methodologies is guiding the protein conformational sampling to achieve electrostatic environments that are underrepresented in the training set. We reserve this development for future work.

\section{pKAl+: Predicting experimental $\mathrm{p} K_{\mathrm{a}}$ values}

The main goal of $\mathrm{p} K_{\mathrm{a}}$ predictors, such as PypKa, is to estimate the macroscopic $\mathrm{p} K_{\mathrm{a}}$ value for the titratable residues using structures (usually experimental ones). Since pKAI aims at reproducing the $\mathrm{p} K_{\text {half }}$ calculated with PypKa at a fraction of the computational cost, it is not expected to outperform the PB-based method in predicting experimental values. When using PB to predict experimental $\mathrm{p} K_{\mathrm{a}} \mathrm{s}$, a higher dielectric constant for the solute is often adopted to compensate for the lack of conformational flexibility in the method and the lack of representativity of the experimental input structure. A similar approach can be implemented in pKAI by introducing a regularization weight to the cost function (pKAI+). This regularization penalizes the magnitude of the $\Delta \mathrm{p} K_{\mathrm{a}}$ prediction. In practice, this procedure biases our estimates towards the $\mathrm{p} K_{\mathrm{a}}$ values in water, similarly to what is done by the increased solute dielectric constant in PB-based approaches. It should be noted that pKAI+ has not been trained on experimental $\mathrm{p} K_{\mathrm{a}}$, but rather on the same training set as $\mathrm{pKAI}$.

To evaluate the performance of our model, we have benchmarked it using a data set of 736 titratable 

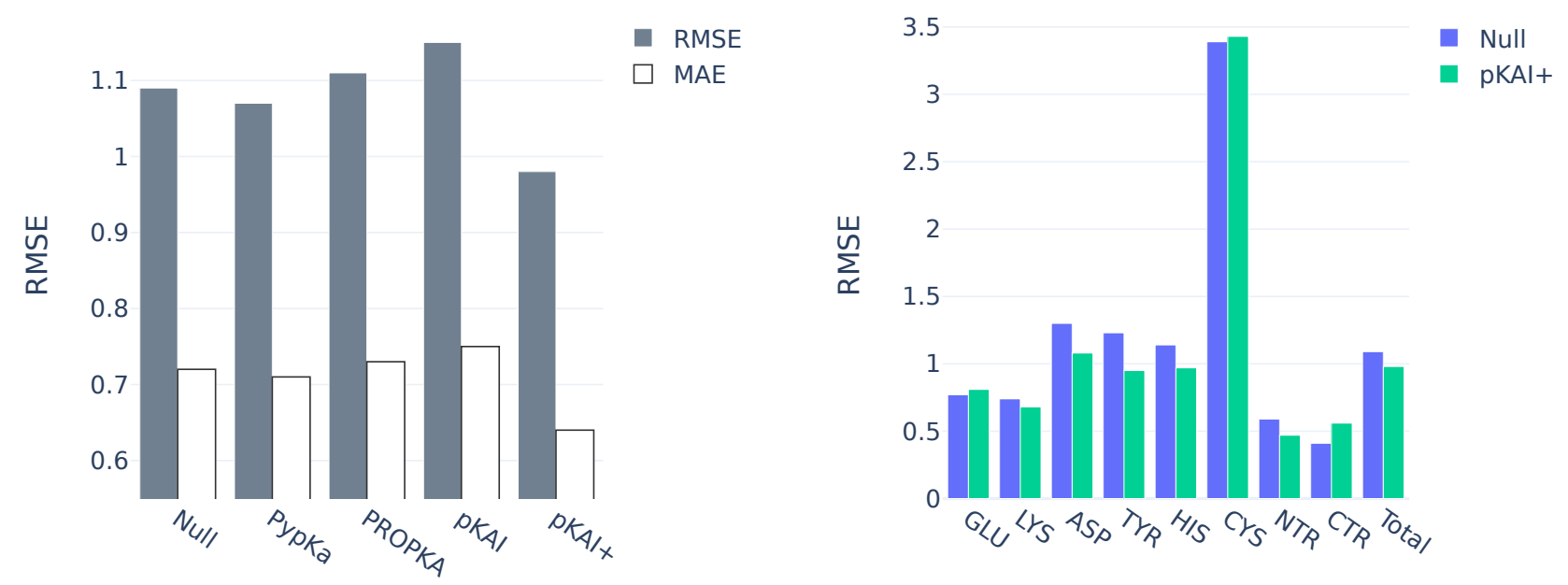

a)

b)
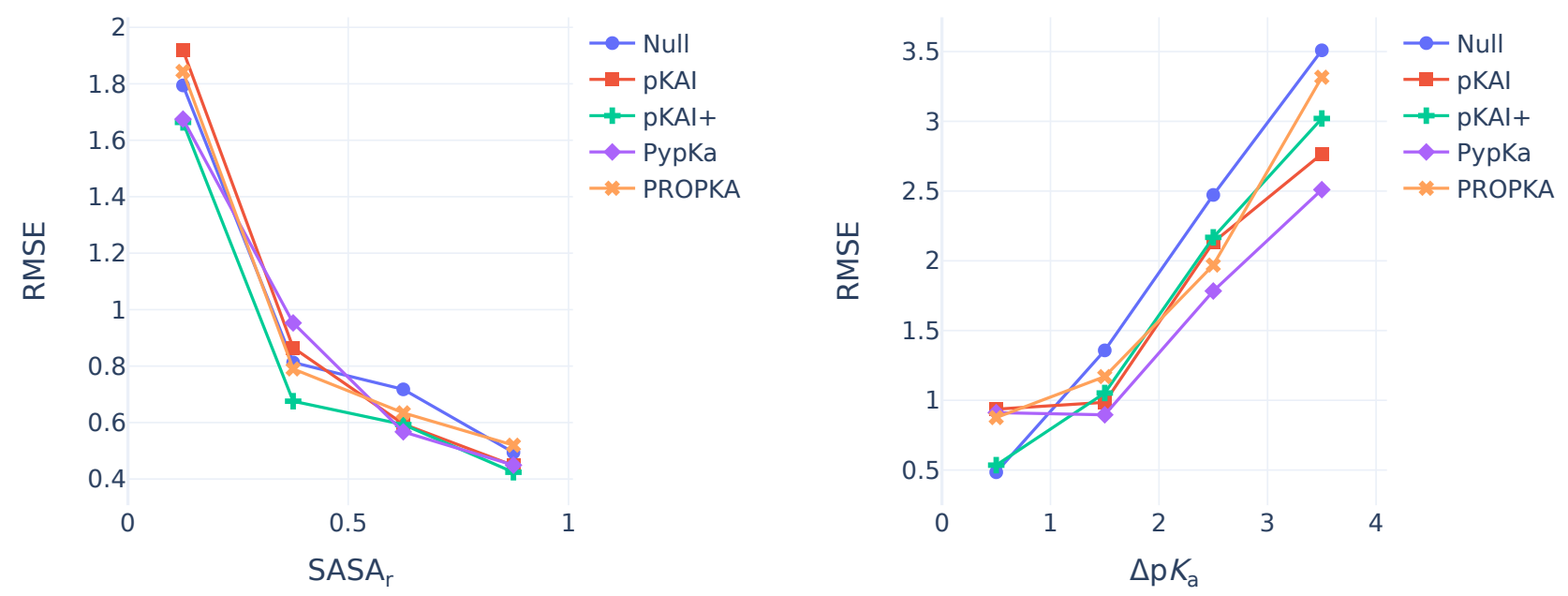

c)

d)

Figure 2. A) Experimental $\mathrm{p} K_{\mathrm{a}}$ benchmark of several methods on a data set of 736 residues from 97 proteins (values shown in Supplementary Table S4). The null model values are the $\mathrm{p} K_{\mathrm{a}}$ values of each amino acid substituted in an alanine pentapetide (Ace-AA-X-AA- $\left.\mathrm{NH}_{2}\right)^{30,31}$. B) Comparison between Null model and pKAI+ performance by residue type. C) pKAI+ performance at predicting experimental $\mathrm{p} K_{\mathrm{a}}$ values dependency on the magnitude of solvent exposure (SASA) of the residues. D) pKAI+ performance dependency on the experimental $\mathrm{p} K_{\mathrm{a}} \operatorname{shift}\left(\Delta \mathrm{p} K_{\mathrm{a}}\right)$. 
residues in 97 proteins with experimentally determined $\mathrm{p} K_{\mathrm{a}}$ values (Figure $2 \mathrm{~A}$ ). Remarkably, pKAI+ (RMSE of 0.98) is able to outperform both PypKa (RMSE of 1.07) and PROPKA (state-of-the art empirical $\mathrm{p} K_{\mathrm{a}}$ predictor, RMSE of 1.11). Furthermore, the improvement over the other methods is significant for most residue types (Figure 2B), and can be quantified using metrics that are more (RMSE, 0.9 quantile) or less (MAE, error percentage under 0.5) sensitive to the presence of outliers (Supplementary Table S3). Cysteine residues are particularly difficult to predict because they naturally occur less frequently and are more buried than all other titratable residues. This leads to an under-representation of these residues in the training set while exhibiting the largest $\mathrm{p} K_{\mathrm{a}}$ shifts. To illustrate the difficulty of this data set, note that some methodologies are not able to improve on the null model (RMSE of 1.09). Hence, the reported deviations are specific to this data set. Even though our benchmark is, to our knowledge, the largest ever used to validate a $\mathrm{p} K_{\mathrm{a}}$ predictor, it is likely still insufficient to quantify the true accuracy of these methods. Nevertheless, pKAI+ was able to improve on the accuracy of the more computationally expensive methodologies while retaining the efficiency of the empirical ones.

Our test set can be divided by solvent exposure (SASA) of the titrating residue. pKAI+ shows comparable RMSE values to PypKa for both the most solvent exposed and buried residues (Figure 2C). Interestingly, it is also able to surpass the PB-based model for partially exposed residues. Notably, pKAI+ only improves the PypKa predictions for $\mathrm{p} K_{\mathrm{a}}$ shifts smaller than $1 \mathrm{p} K$ unit (Figure 2D). This indicates that $\mathrm{pKAI}+$ corrects the $\mathrm{p} K_{\mathrm{a}}$ values of partially exposed residues which are establishing nonrepresentative interactions in the experimental structure. Since there is a large number of residues with these characteristics in the test $\operatorname{set}^{28}$, the overall performance improvement is significant (Supplementary Table S4).

\section{Model Explainability}

The main driving force for $\mathrm{p} K_{\mathrm{a}}$ shifts in proteins is electrostatic in nature. In our model, each atom of the environment represents the contribution of a chemical group or part of a residue. This individual contribution towards the final $\Delta \mathrm{p} K_{\mathrm{a}}$ prediction can be estimated (see XAI in the Methods section for further details) and it is shown in Figure 3A. Remarkably, although our model has been given no information about atomic charges, it assigns contributions that are in agreement with the expected overall charge of the atom class. Cationic amine groups (NZ_LYS; NH_ARG; NE_ARG; NE2_HIS) are clearly assigned positive scores (i.e. destabilize the protonation of the titratable residue) and are easily distinguishable from the anionic carbonyl groups (O_COOH from Glu, Asp and C-termini residues). These scores provide a general insight into the network's interpretation of each atom and should not be used for more quantitative analysis. Since the atom score is an averaged measure across the test set, an imbalance of closely interacting atoms of a specific class can dramatically skew its median contribution. 
Hydrogen bonds are one of the strongest interactions found in proteins, and, as such, their proper description is crucial to obtain accurate $\mathrm{p} K_{\mathrm{a}}$ predictions. By comparing Figure $3 \mathrm{~A}$ and Figure 3B we can observe marked differences between the atom scores at close proximity and those farther away from the titrating residue. For example, the average score of the very abundant classes of primary amines $(\mathrm{N}$; N_AMIDE) and carbonyl groups (O; O_AMIDE) is greatly diminished when compared to their short-range contributions, where these become hydrogen donors and acceptors, respectively. The anionic Tyr residue is perceived to have an overall negative contribution, except when it is close to another titratable residue; in this case there seems to be no preferred state as it can act both as a donor and as an acceptor - like any titratable residue. On the other hand, the contribution of neutral non-titrating alcohol groups (OG_SER; OG1_THR) is almost exclusively attributed to their potential to form hydrogen bonds at short range. Overall, the model is able to capture an astonishing amount of details concerning the physics underlying $\mathrm{p} K_{\mathrm{a}}$ shifts.

Beyond the general understanding shown before, hydrogen bond contributions are hard to account for, compared to other interactions. As shown in Figure $3 \mathrm{C}$ the closer another residue (blue curve) is to the titrating one, the harder for the model is to correctly describe their interaction. The difficulty of the prediction increases dramatically at the typical distance of hydrogen bonds (2.5-3.2@). This is even more marked if one considers interactions established between two titratable residues (red curve). In this case, the network has to solve for the $\mathrm{p} K_{\mathrm{a}}$ of both residues simultaneously, and in many instances it is unable to do so. Hence, predicting the contribution of the remaining environment is easier than that of a single hydrogen bond. This is illustrated in Figure 3D, in which the agreement with the physics-based method is much higher when the closest atom is removed from the structure rather than when it is kept in its original position. Although many other profiles can be observed (Supplementary Figure S3), this trend is generally conserved. Considering that the model did not receive explicit information about hydrogen bonds, it is quite remarkable that it was able to correlate this type of interaction with larger $\mathrm{p} K_{\mathrm{a}}$ shifts.

Solvent exposure is another property that is usually a key contributor to $\mathrm{p} K_{\mathrm{a}}$ shifts. The models are trained without explicit knowledge of the 3D structure of the protein, and deprived of information regarding carbon atoms. Nevertheless, they seem to learn about the solvent exposure contribution. We compared the correlations (Pearson correlation coefficient $r$ and Spearman's rank correlation coefficient $\rho$ ) between the calculated solvent exposure (SASA) and the $\mathrm{p} K_{\mathrm{a}}$ shifts over the entire test data set. Using the known $\Delta \mathrm{p} K_{\mathrm{a}}$, we obtained $r_{\Delta \mathrm{pka}}=-0.68 ; \rho_{\Delta \mathrm{pka}}=-0.60$, while using the predicted $\Delta \mathrm{p} K_{\mathrm{a}}$, we got $r_{\text {pred }}=-0.66 ; \rho_{\text {pred }}=-0.62$. The similarity between these values indicates that the model has learned the correct correlation between SASA and the $\mathrm{p} K_{\mathrm{a}}$ shift. Additionally, we trained a model where we provided SASA as an additional input and observed no performance improvement (data not shown). 


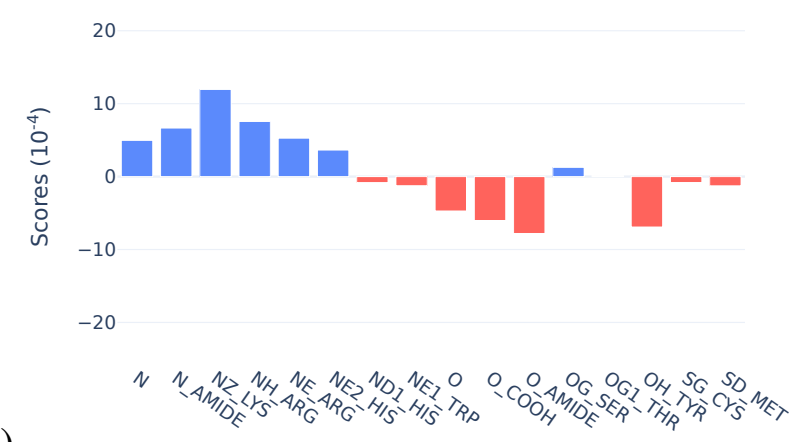

a)

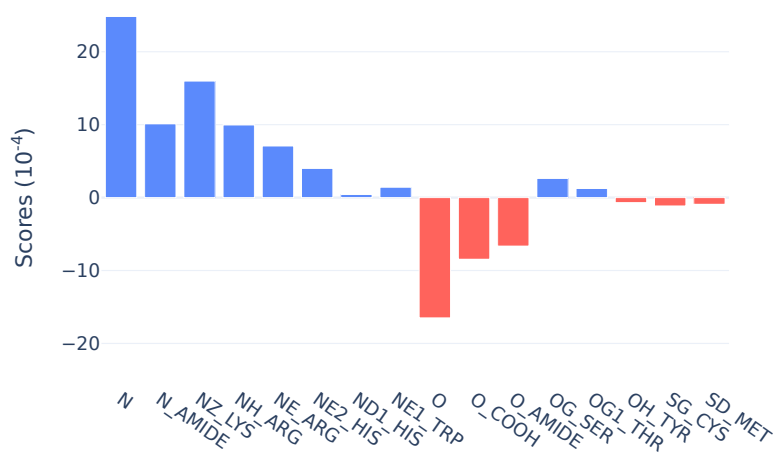

b)
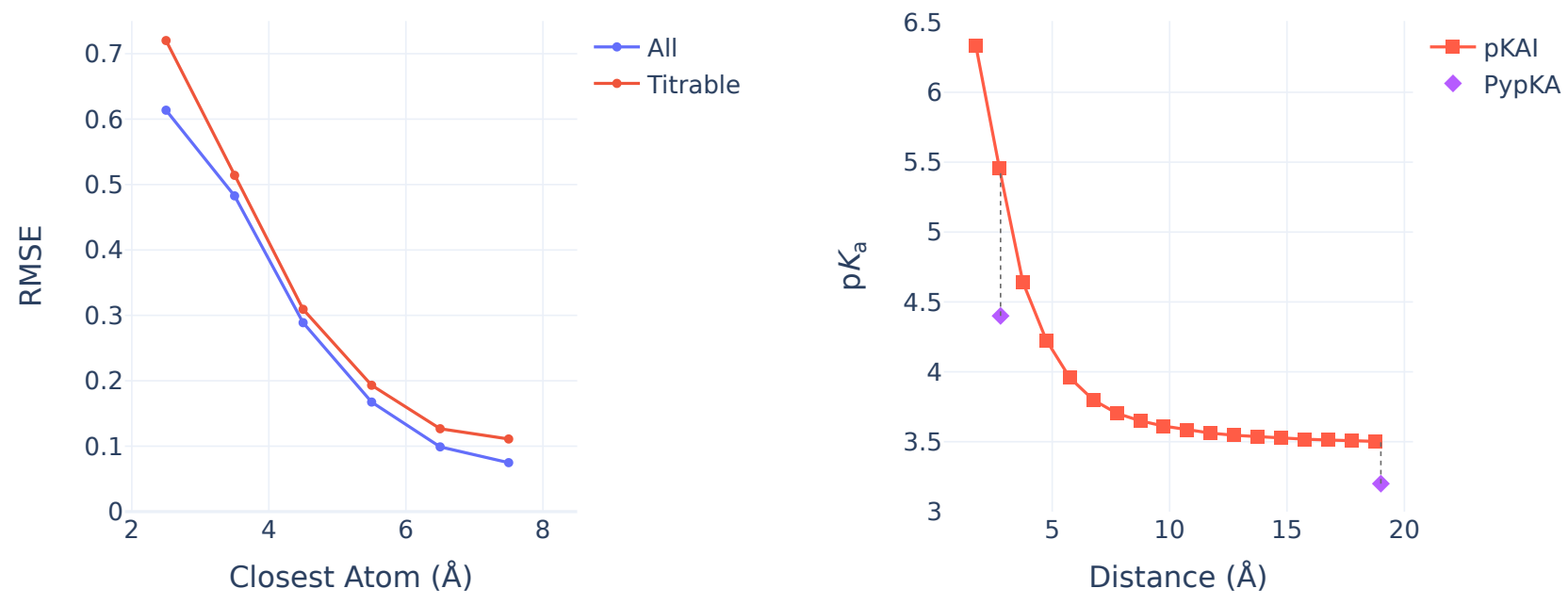

c)

d)

Figure 3. A) Charge scores attributed by pKAI to all considered input atoms classes (S5). B) Charges scores of atoms closer than $6 \AA$. C) Closest atom influence on pKAI performance. D) Impact of changing the distance of the closest atom on pKAI predictions of residue TYR-315 from structure 2BJU. For reference, we have included PypKa predictions of the same residue in the state presented in the experimental structure (closest distance $2.8 \AA$ ) and in a modified structure in which the closest atom is absent. 
Finally, it is worth mentioning that XAI analysis was a driving factor in the development of pKAI. In fact, the importance that the model assigns to each atom class (similar to Figure 3A) was pivotal to select the final set of atom classes aimed at describing the surrounding environment residues.

\section{Discussion}

We have introduced pKAI and pKAI+, two deep learning models to predict theoretical and experimental $\Delta \mathrm{p} K_{\mathrm{a}}$ values, respectively. pKAI offers unprecedented efficiency, exhibiting a remarkable trade-off between accuracy and computational speed, its performance rivaling that of CE-based methods, such as PypKa. pKAI could be used as a replacement for such methods, especially when dealing with large proteins or applications requiring multiple $\mathrm{CE}$ calculations, like constant-pH MD simulations ${ }^{16-20}$. Considering the latest advances in sequence to structure predictions ${ }^{32}$, faster methods, such as pKAI, will likely be of use as exponentially more structures become available. Furthermore, when optimizing new structures for binding to specific targets (e.g. design of enzymes and/or antibodies), it is vital to have an accurate prediction of the protonation states.

While we strive for optimal accuracy, we are aware that many applications will only require a binary decision (hence a qualitative prediction of $\mathrm{p} K_{\mathrm{a}}$ shifts would be sufficient). For example, when selecting the most likely protonation state of a protein, one only needs to predict whether each $\mathrm{p} K_{\mathrm{a}}$ is larger or smaller than the $\mathrm{pH}$ value of interest. At the physiological $\mathrm{pH}$ range, $\mathrm{pKAI}$ achieves an astounding accuracy of $99.4 \%$ (see Supplementary Figure S2). Several other applications only require an estimation of the proton binding affinity using a fixed conformation. This quantity, termed $\mathrm{p} K_{\text {half }}$, renders a good prediction of the macroscopic $\mathrm{p} K_{\mathrm{a}}$ when averaged over a representative ensemble of conformations. From $\mathrm{p} K_{\text {half }}$ values, the most abundant/representative protonation states for a particular conformation can be calculated, improving the realism of methods such as molecular dynamics ${ }^{16-20}$ and molecular docking ${ }^{33}$. However, in a conformational ensemble, there are always many representative protonation states which differ significantly from the one calculated using the macroscopic $\mathrm{p} K_{\mathrm{a}}$ values. Therefore, coupling $\mathrm{p} K_{\text {half }}$ calculations with conformational sampling techniques is very appealing in theory but difficult in practice, due to their computational cost. By using pKAI instead of PypKa (or any other PB-based method), one would drastically decrease the computational overhead (up to $1000 \times$ ).

pKAI does not handle all residues with the same performance. Difficult cases are caused by low representation in the training set, low solvent exposure, and/or close-by residues providing $\mathrm{H}$ bond interactions. These peculiar environments usually present a high $\Delta \mathrm{p} K_{\mathrm{a}}$ which is not handled very well by the method. One clear way to improve our models would therefore be to introduce more training examples; 
we showed in Figure 1D that our overall RMSE shows constant decrease with addition of more training data. Furthermore, the inclusion of more training data with rare environments would definitely enhance performance. To better handle interactions with neighboring titratable groups, a change of environment encoding would be needed. One approach to be explored in future work would be to represent the whole protein as a graph, and use newly developed graph neural networks algorithms to learn the $\Delta \mathrm{p} K_{\mathrm{a}}$ values.

Although pKAI excels at predicting $\mathrm{p} K_{\text {half }}$ values, its performance is modest when estimating experimental $\mathrm{p} K_{\mathrm{a}}$ values. Inspired by the observation that increasing the dielectric constant in PB-based methods improves their agreement with experimental results, we have introduced a regularization parameter into the cost function. Similar to the dielectric constant, this regularization weight biases all predictions towards the residue's $\mathrm{p} K_{\mathrm{a}}$ values in water. The new model, $\mathrm{pKAI}+$, outperforms all methods tested in this work, including PypKa which was used to create the training set. However, this improvement, while significant for partially exposed residues which would otherwise exhibit overestimated $\mathrm{p} K_{\mathrm{a}}$ shifts, penalizes the accuracy of more shifted residues.

With $\mathrm{pKAI}(+)$, we are introducing the first deep learning-based predictors of $\mathrm{p} K_{\mathrm{a}}$ shifts in proteins. The unique combination of speed and accuracy afforded by our models represents a paradigm shift in $\mathrm{p} K_{\mathrm{a}}$ predictions. $\mathrm{pKAI}(+)$ paves the way for accurate estimations of macroscopic $\mathrm{p} K_{\mathrm{a}}$ values from ensemble calculations of $\mathrm{p} K_{\text {half }}$ values, overcoming previous computational limits. By design, the models were trained using a very simplified view of the surroundings of the titratable group, ignoring all carbon and hydrogen atoms, and limiting different atom types. This design choice allowed for the models to stay small and fast. Explainability methods confirmed that this input information was enough for the model to capture crucial features such as electrostatics, solvent exposure, and environment contributions. The models' initial success introduces several opportunities for further research, including problem encoding, accounting for conformational flexibility, and adding further target properties that could be of interest for other applications.

\section{Methods}

\section{Data set}

To train our DL models, we used a large publicly available data set of estimated $\mathrm{p} K$ values - the pKPDB database $^{28}$. This data set of $\sim 3 \mathrm{M} \mathrm{p} K_{\mathrm{a}}$ values was created by running the PypKa tool with default parameters ${ }^{6}$ over all the protein structures deposited on the Protein Data Bank. The target values to be fitted by our model are theoretical $\mathrm{p} K_{\text {half }}$ values estimated with a PB-based method. This implies that pKAI will inherit the assumptions and limitations of this class of predictors. Our approach contrasts with 
the one usually adopted for training empirical predictors, which entails using experimental values to fit the model's parameters. The main advantage of this novel approach is that we can train models with significantly more parameters, such as deep learning ones, since there is now a much larger abundance of training data. As a comparison, in PROPKA3 only 85 experimental values of aspartate and glutamate residues were used to fit 6 parameters ${ }^{5}$. This bigger data set translates into more diversity in terms of protein and residue types and, more importantly, a wider variety of residue environments. Furthermore, the relation between a structure and our target property is deterministic, contrary to that of experimental $\mathrm{p} K_{\mathrm{a}}$ values, which suffers from the lack of entropic information.

The ultimate goal of these methods is to accurately predict experimental $\mathrm{p} K_{\mathrm{a}}$ values and thus, we have assessed the model's performance with $\sim 750$ experimental $\mathrm{p} K_{\mathrm{a}}$ values taken from the PKAD database $^{34}$. We compare our experimental results with a null model (attributing to each titratable group the corresponding $\mathrm{p} K_{\mathrm{a}}$ value in water), PypKa (the method used to generate the training set) and PROPKA with default settings (the empirical method of reference).

Before training our models on our data set, we applied a curated data split (see Figure 4A) to ensure that the training, validation, and test sets did not contain proteins with a high degree of similarity. First, we randomly selected 3.000 proteins from the full data set of $\sim 120 \mathrm{k}$ proteins as our holdout test set of theoretical $\mathrm{p} K_{\mathrm{a}}$ values. The program mmseqs ${ }^{35}$ was then used to exclude all proteins containing at least one chain similar to any of the chains found either in the experimental or in the theoretical test sets. Chains were considered to be similar if they presented sequence identity over $90 \%$. From the remaining set of proteins, 3.000 more were randomly assigned to the validation set while the rest became the training set. Finally, we have excluded similar proteins to those of the validation set from the training set.

\section{Model architecture and implementation}

pKAI is implemented and trained using PyTorch v1.9.0 $0^{36}$ and PyTorch Lightning v1.2.10 37 The model has a simple architecture comprised of 3 fully-connected hidden layers in a pyramidal configuration fitted to the $\mathrm{p} K_{\mathrm{a}}$ shifts of titratable amino acids (Figure $4 \mathrm{~B}$ ).

The encoding of the environment of each titratable residue has been simplified to retain only the most important electrostatic descriptors (Figure 4C). Considering the decay rate of the electrostatic potential, we decided to truncate the contributions to the environment of a residue by applying a cutoff of $15 \AA$ around the labile atom(s) of the titratable residue. In practice, this cutoff is slightly smaller for some residue environments as the necessary input layer size normalization resulted in the truncation of the closest 250 atoms. A further approximation was made by considering only highly charged atoms and assuming they 


\begin{tabular}{lll}
\hline Split & Proteins & $\begin{array}{l}\mathrm{p} K \text { values } \\
\text { (thousands) }\end{array}$ \\
\hline All & 116203 & 12600.1 \\
Train & 56778 & 6345.4 \\
Validation & 3000 & 322.4 \\
Test Theor. & 3000 & 325.3 \\
Test Exp. & 97 & 736 \\
\hline
\end{tabular}

a)

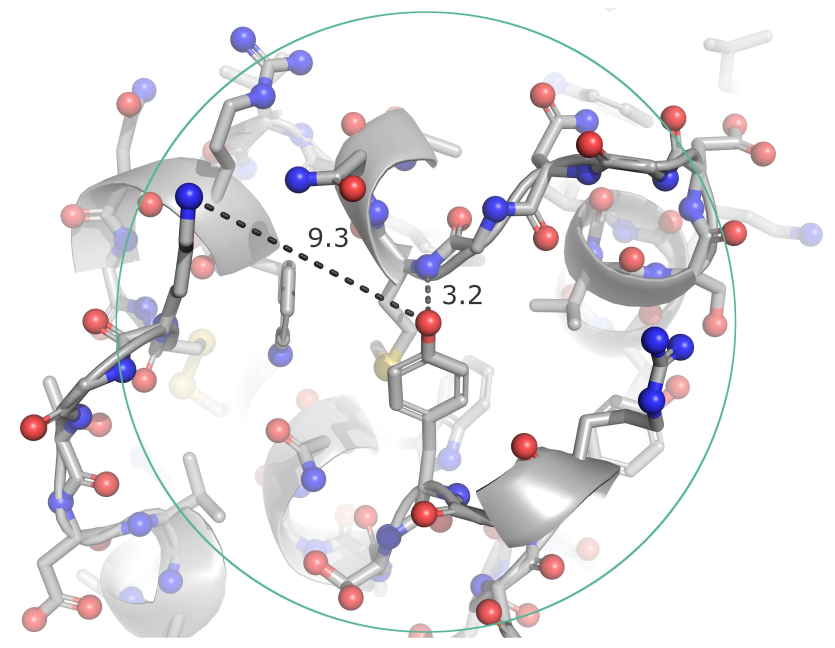

c)

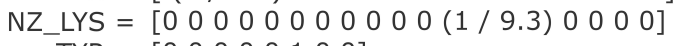
TYR = $\left[\begin{array}{llllllll}0 & 0 & 0 & 0 & 0 & 1 & 0 & 0\end{array}\right]$

Figure 4. A) Overview of the data split. B) pKAI model architecture. C) Illustration of the titratable amino acid environment encoding. Only Nitrogen, Oxygen and Sulfur atoms (shown as spheres) within a $15 \AA$ A cutoff (green circle) are included while all carbon (shown as sticks) and hydrogens (omitted) are ignored. The included atoms are represented by the inverse of their distance to the titratable residue in a OHE vector featuring 16 categories of atom classes (Table S5). The titratable residue is represented by a OHE vector comprised of 8 classes. D) pKAI+ regularization weight performance test. 
For each input atom feature $\widehat{a}=\left(a, r_{a}\right)$, where $a$ indicates the atom type and $r_{a}$ the corresponding distance to the liable atom(s) of the titrating residue, we compute the corresponding attribution $I(\widehat{a})$ with the Integrated Gradients (IG) algorithm, ${ }^{40}$ as implemented in the shap package ${ }^{41} . I(\widehat{a})$ measures the sensitivity of the network output with respect to changes in the input $\widehat{a}$. A large absolute value of $I(\widehat{a})$

are the ones that contribute the most to $\mathrm{p} K_{\mathrm{a}}$ shifts. This simplification can be slightly compensated by using atom classes instead of charges or element names as they implicitly provide information about adjacent atoms. The atoms were one-hot encoded (OHE) and, in order to reduce the input layer size, chemically similar atoms were assigned to the same category (Table S5). While carboxylic oxygen atoms (C-termini OXT; aspartates OD1 and OD2; glutamates OE1 and OE2) and primary amine atoms (arginines $\mathrm{NH} 1$ and NH2) atoms have been merged, others with similar names but different chemical properties were separated (glutamines OE1 and NE2 from glutamates OE1 and histidines NE2, asparagines OD1 from aspartates OD1; main chain $\mathrm{N}$ from $\mathrm{N}$-termini $\mathrm{N}$ ).

The final 4008-sized input layer consisted of 250 atoms represented by 16 categories OHE classes concatenated to an 8-dimension OHE vector corresponding to the titrating amino acid. The atom's OHE was multiplied by its reciprocal distance to the titrating residues so that this valuable information could be included without increasing the size of the input layer.

pKAI is freely available as a python module that can be installed via pip. The source code can be found at https://github.com/bayer-science-for-a-better-life/pKAI.

\section{Training}

Training was performed with mini-batches of 256 examples and the Adam optimizer ${ }^{38}$ with a learning rate of $1 e^{-6}$ and weight decay of $1 e^{-4}$. Dropout regularization was applied to all fully-connected layers

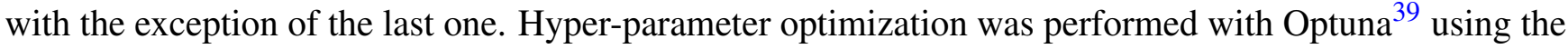
performance in the validation set. Training these models takes approximately 10 minutes on an NVIDIA Tesla M40 24GB, using 16bit precision and an early stopping strategy on the minimization of the cost function with a delta of $1 e^{-3}$ and patience of 5 steps.

The pKAI model was trained on an MSE cost function while for the pKAI+ we have added a regularization parameter to penalize $\Delta \mathrm{p} K_{\mathrm{a}}$ predictions. Different regularization weights were tested to check for possible overfitting (Figure 4D). While a 50\% weighting was deemed the best for this particular validation set, any value between $40 \%$ and $70 \%$ would lead to similar accuracy improvements.

\section{XAI Methods}


indicates that the network assigns high importance to this feature, while the sign of $I(\widehat{a})$ indicates whether the feature contributes positively or negatively to the output. Given that the most important contributions to the $\Delta \mathrm{p} K_{\mathrm{a}}$ are of electrostatic nature, one can try to explain the model inferred charges for all atom classes by computing the distant-independent score

$$
C(a)=\mathbb{E}\left[r_{a}^{-1} I_{-}(\widehat{a})\right]-\mathbb{E}\left[r_{a}^{-1} I_{+}(\widehat{a})\right]
$$

where

$$
I_{ \pm}(\widehat{a})=\left\{\begin{array}{ll}
I(\widehat{a}) & \text { if } \pm I(\widehat{a})>0 \\
0 & \text { otherwise }
\end{array} .\right.
$$

The expectation value here is over a large subset (10000 samples) of the test set. The sign of $C(a)$ in equation 1 resembles the charge that the network, on average, assigns to a given atom type. For example, if an atom class is being perceived by the model as contributing negatively to the $\Delta \mathrm{p} K_{\mathrm{a}}$ (positive $C(a)$ ), this would mean that the network learned that this particular atom stabilizes the deprotonated state, which is characteristic of positively charged groups.

The solvent accessible surface area shown in Table S1 and in the XAI subsection have been taken from $\mathrm{pKPDB}^{28}$.

\section{References}

1. Warshel, A. \& Åqvist, J. Electrostatic energy and macromolecular function. Annu. Rev. Biophys. Biophys. Chem. 20, 267-298 (1991).

2. Kim, J., Mao, J. \& Gunner, M. Are acidic and basic groups in buried proteins predicted to be ionized? J. Mol. Biol. 348, 1283-1298 (2005).

3. Anandakrishnan, R., Aguilar, B. \& Onufriev, A. V. H++ 3.0: automating pK prediction and the preparation of biomolecular structures for atomistic molecular modeling and simulations. Nucleic Acids Res. 40, W537-W541 (2012).

4. Georgescu, R. E., Alexov, E. G. \& Gunner, M. R. Combining conformational flexibility and continuum electrostatics for calculating pKas in proteins. Biophys. J. 83, 1731 - 1748, DOI: https://doi.org/10. 1016/S0006-3495(02)73940-4 (2002).

5. Olsson, M. H., Søndergaard, C. R., Rostkowski, M. \& Jensen, J. H. PROPKA3: consistent treatment of internal and surface residues in empirical $\mathrm{p} K_{\mathrm{a}}$ predictions. J. Chem. Theory Comput. 7, 525-537 (2011). 
6. Reis, P. B. P. S., Vila-Viçosa, D., Rocchia, W. \& Machuqueiro, M. PypKa: A flexible python module for poisson-boltzmann-based $\mathrm{p} K_{\mathrm{a}}$ calculations. J. Chem. Inf. Model. 60, 4442-4448, DOI: 10.1021/acs.jcim.0c00718 (2020). PMID: 32857502.

7. Wang, L., Zhang, M. \& Alexov, E. DelPhiPKa web server: predicting $\mathrm{p} K_{\mathrm{a}}$ of proteins, rnas and dnas. Bioinformatics 32, 614-615 (2016).

8. Schutz, C. N. \& Warshel, A. What are the dielectric "constants" of proteins and how to validate electrostatic models? Proteins: Struct. Funct. Genet. 44, 400-417 (2001).

9. Voges, D. \& Karshikoff, A. A model of a local dielectric constant in proteins. J. Chem. Phys. 108, 2219-2227, DOI: 10.1063/1.475602 (1998). https://doi.org/10.1063/1.475602.

10. Demchuk, E. \& Wade, R. C. Improving the continuum dielectric approach to calculating pKas of ionizable groups in proteins. J. Phys. Chem. 100, 17373-17387, DOI: 10.1021/jp960111d (1996). https://doi.org/10.1021/jp960111d.

11. Rocchia, W., Alexov, E. \& Honig, B. Extending the applicability of the nonlinear Poisson-Boltzmann equation: multiple dielectric constants and multivalent ions. J. Phys. Chem. B 105, 6507-6514 (2001).

12. Li, L., Li, C., Zhang, Z. \& Alexov, E. On the dielectric "constant" of proteins: Smooth dielectric function for macromolecular modeling and its implementation in delphi. J. Chem. Theory Comput. 9, 2126-2136, DOI: 10.1021/ct400065j (2013). PMID: 23585741, https://doi.org/10.1021/ct400065j.

13. Beroza, P. \& Case, D. A. Including side chain flexibility in continuum electrostatic calculations of protein titration. J. Phys. Chem. 100, 20156-20163 (1996).

14. Nielsen, J. E. \& Vriend, G. Optimizing the hydrogen-bond network in Poisson-Boltzmann equationbased $\mathrm{p} K_{\mathrm{a}}$ calculations. Proteins Struct. Funct. Bioinf. 43, 403-412, DOI: 10.1002/prot.1053 (2001). https://onlinelibrary.wiley.com/doi/pdf/10.1002/prot.1053.

15. Baptista, A. M. \& Soares, C. M. Some theoretical and computational aspects of the inclusion of proton isomerism in the protonation equilibrium of proteins. J. Phys. Chem. B 105, 293-309 (2001).

16. Baptista, A. M., Teixeira, V. H. \& Soares, C. M. Constant-pH molecular dynamics using stochastic titration. J. Chem. Phys. 117, 4184-4200 (2002).

17. Mongan, J., Case, D. A. \& McCammon, J. A. Constant pH molecular dynamics in generalized Born implicit solvent. J. Comput. Chem. 25, 2038-2048 (2004).

18. Khandogin, J. \& Brooks III, C. L. Toward the accurate first-principles prediction of ionization equilibria in proteins. Biochemistry-US 45, 9363-9373 (2006). 
19. Swails, J. M. \& Roitberg, A. E. Enhancing conformation and protonation state sampling of hen egg white lysozyme using $\mathrm{pH}$ replica exchange molecular dynamics. J. Chem. Theory Comput. 8, 4393-4404 (2012).

20. Vila-Viçosa, D., Reis, P. B. P. S., Baptista, A. M., Oostenbrink, C. \& Machuqueiro, M. A pH replica exchange scheme in the stochastic titration constant-pH MD method. J. Chem. Theory Comput. 15, 3108-3116, DOI: 10.1021/acs.jctc.9b00030 (2019).

21. Teixeira, V. H., Vila-Viçosa, D., Reis, P. B. \& Machuqueiro, M. pK $K_{\mathrm{a}}$ values of titrable amino acids at the water/membrane interface. J. Chem. Theory Comput. 12, 930-934 (2016).

22. Vila-Viçosa, D., Campos, S. R. R., Baptista, A. M. \& Machuqueiro, M. Reversibility of prion misfolding: insights from constant-pH molecular dynamics simulations. J. Phys. Chem. B 116, 8812-8821 (2012).

23. Morrow, B. H., Koenig, P. H. \& Shen, J. K. Atomistic simulations of pH-dependent self-assembly of micelle and bilayer from fatty acids. J. Chem. Phys. 137, 194902-194902 (2012).

24. Swails, J. M. et al. pH-dependent mechanism of nitric oxide release in nitrophorins 2 and 4. J. Phys. Chem. B 113, 1192-1201, DOI: 10.1021/jp806906x (2009).

25. Reis, P. B., Vila-Viçosa, D., Campos, S. R., Baptista, A. M. \& Machuqueiro, M. Role of counterions in constant-pH molecular dynamics simulations of PAMAM dendrimers. ACS Omega 3, 2001-2009 (2018).

26. Stanton, C. L. \& Houk, K. N. Benchmarking pKa prediction methods for residues in proteins. J. Chem. Theory Comput. 4, 951-966, DOI: 10.1021/ct8000014 (2008). PMID: 26621236.

27. Lee, A. C. \& Crippen, G. M. Predicting pKa. J. Chem. Inf. Model. 49, 2013-2033, DOI: 10.1021/ ci900209w (2009). PMID: 19702243.

28. Reis, P. B. P. S., Clevert, D.-A. \& Machuqueiro, M. pKPDB: a protein data bank extension database of pKa and pI theoretical values. Bioinformatics DOI: 10.1093/bioinformatics/btab518 (2021).

29. Machuqueiro, M. \& Baptista, A. M. Is the prediction of $\mathrm{p} K_{\mathrm{a}}$ values by constant-pH molecular dynamics being hindered by inherited problems? Proteins Struct. Funct. Bioinf. 79, 3437-3447 (2011).

30. Thurlkill, R. L., Grimsley, G. R., Scholtz, J. M. \& Pace, C. N. pK values of the ionizable groups of proteins. Protein Sci. 15, 1214-1218 (2006).

31. Grimsley, G. R., Scholtz, J. M. \& Pace, C. N. A summary of the measured $\mathrm{p} K$ values of the ionizable groups in folded proteins. Protein Sci. 18, 247-251 (2009). 
32. Jumper, J. et al. Highly accurate protein structure prediction with alphafold. Nature 596, 583-589, DOI: 10.1038/s41586-021-03819-2 (2021).

33. Onufriev, A. V. \& Alexov, E. Protonation and pK changes in protein-ligand binding. Q. Rev. Biophys. 46, 181-209, DOI: 10.1017/S0033583513000024 (2013).

34. Pahari, S., Sun, L. \& Alexov, E. PKAD: a database of experimentally measured $\mathrm{p} K_{\mathrm{a}}$ values of ionizable groups in proteins. Database 2019 (2019).

35. Mirdita, M., Steinegger, M. \& Söding, J. MMseqs2 desktop and local web server app for fast, interactive sequence searches. Bioinformatics 35, 2856-2858, DOI: 10.1093/bioinformatics/bty1057 (2019).

36. Paszke, A. et al. Pytorch: An imperative style, high-performance deep learning library. In Wallach, $H$. et al. (eds.) Advances in Neural Information Processing Systems 32, 8024-8035 (Curran Associates, Inc., 2019).

37. Falcon, W. Pytorch lightning. GitHub. Note: https://github.com/PyTorchLightning/pytorch-lightning 3 (2019).

38. Kingma, D. P. \& Ba, J. L. Adam: A method for stochastic gradient descent. In ICLR: International Conference on Learning Representations, 1-15 (2015).

39. Akiba, T., Sano, S., Yanase, T., Ohta, T. \& Koyama, M. Optuna: A next-generation hyperparameter optimization framework. In Proceedings of the 25rd ACM SIGKDD International Conference on Knowledge Discovery and Data Mining (2019).

40. Sundararajan, M., Taly, A. \& Yan, Q. Axiomatic attribution for deep networks (2017). 1703.01365.

41. Lundberg, S. M. \& Lee, S.-I. A unified approach to interpreting model predictions. In Guyon, I. et al. (eds.) Advances in Neural Information Processing Systems 30, 4765-4774 (Curran Associates, Inc., 2017).

\section{Acknowledgements}

We would like to thank Paulo Martel and Diogo Vila-Viçosa for the fruitful discussions as well as the attendees of the Protein Electrostatics meeting (www.proteinelectrostatics.org). We thank Artemi Bendandi for proofreading the manuscript.

PR and MM acknowledge financial support from FCT: SFRH/BD/136226/2018, CEECIND/02300/2017, UIDB/04046/2020, and UIDP/04046/2020; and the EGI infrastructure: IN2P3-IRES, and NCG-INGRID- 
PT. MB and FM acknowledge funding from the Bayer AG Life Science Collaboration ("Explainable $\left.A I^{\prime \prime}\right)$.

\section{Author Contributions}

PR, FM and MB planned the experiments. PR performed the implementation of pKAI and analysis. MB performed the explainability experiments. PR, FM and MM conceived and designed pKAI. PR, MM, FM and MB wrote the paper with the help of DC and WR. 


\section{Supplementary Files}

This is a list of supplementary files associated with this preprint. Click to download.

- si.pdf 